Copyright (C 2014 IEEE. Personal use of this material is permitted. Permission from IEEE must be obtained for all other uses, in any current or future media, including reprinting/republishing this material for advertising or promotional purposes, creating new collective works, for resale or redistribution to servers or lists, or reuse of any copyrighted component of this work in other works. 


\title{
Tongue-Supported Human-Computer Interaction Systems: A Review
}

\author{
Masood Mehmood Khan, Hammad I. Sherazi, and Rohan Quain
}

\begin{abstract}
The tongue can substitute human sensory systems and has been used as a medium of input to help impaired patients communicate with the world. Innovative techniques have been employed to realize tongue movement, sense its position and exploit tongue dexterity, in order to achieve Tongue Supported Human Computer Interaction (TSHCI). This paper examines various approaches of using tongue dexterousness in TSHCI systems and then introduces two infrared signal supported minimally-invasive TSHCI systems developed at Curtin University. Methods of sensing tongue movement and position are especially discussed and depending on the employed methods, TSHCI systems are categorized as either invasive or minimally-invasive. A set of system usability criteria is proposed to help build more effective TSHCI systems in future.
\end{abstract}

\section{INTRODUCTION}

The tongue has been used in tactile vision substitution systems for form-perception [1]. The missing sensory component to the sensory motor loop was also provided using the tongue $[2,3]$. In order to improve human balance and prevent people from non-voluntary fall, the tongue has also been used in biofeedback systems [4]. Building upon these applications, recent Human-Computer Interaction (HCI) literature provides insight into methods, design related problems and implementation approaches developed to help improve quality of life for critically ill patients. The recent HCI systems for enabling impaired patients interact with men and machines differ in terms of design and interaction approaches. The HCI systems developed during the last ten years have exploited many of the available input modalities such as; voice commands, brain signals, heart beats, eye movement, head movements, and tongue $[5,6]$. Interestingly, some systems have also employed a combination of two or more of these input modalities [5-7]. Tongue-activated interaction systems are considered promising and more acceptable, especially for quadriplegic, impaired and critically injured people [8-12].

A particular class of HCI systems, the Tongue-Supported Human Computer Interaction (TSHCI) systems, can be useful for people with serious communication limitations caused by spinal cord related injuries, brain damage, or a non-functional sensory-motor system. For their limited demography, scope and applicability, TSHCI systems have received less attention

Masood Mehmood Khan is with Curtin University, Department of Mechanical Engineering, Perth Western Australia 6012 (phone: +61 89266 9205; e-mail: Masood.Khan@curtin.edu.au).

Hammad I. Sherazi is with the National University of Science \& Technology, Karachi, Pakistan, h.sherazi@pnec.nust.edu.pk

Rohan Quain is a Mechatronic Engineering graduate of Curtin University (phone: +61 0419659221; e-mail: rohanquain@gmail.com). compared with HCI systems that utilize voice commands, brain signals, eye tracking, body and/or head movement signals and traditional input. Consequently, TSHCI systems are far from being commercially available for use in real life situations. Major limitations of TSHCI systems can be attributed to the following issues.

Firstly, it is difficult to understand and exploit the mechanical and functional characteristics of the tongue for designing a universally acceptable and appropriate TSHCI system. Especially, tongue dexterousness of a particular patient-demography is difficult to determine, analyze and exploit. Secondly, methods of monitoring tongue movement and position are difficult to develop and use in TSHCI systems. Thirdly, data and standards on various aspects of sensing and control schemes used in TSHCI systems is not available yet as the systems haven't been tested in real life situations. Lastly, a major problem associated with the TSHCI systems is that some kind of an invasive procedure is needed for enabling tongue movement realization and position sensing in TSHCI systems. Almost all methods used for determining tongue movement/location in major TSHCI systems (developed during the last decade or so) would require a patient to go through an expensive yet painful process [9-18]. A great majority of the existing TSHCI systems would require physical attachment of sensing media with mouth and/or tongue. Thus, TSHCI systems remain far from being usable and acceptable.

In order to address the usability and acceptability issues of TSHCI systems, new tongue movement/location realization methods need to be developed and tested. Attempts were made at Curtin University to design and implement TSHCI systems that wouldn't require a highly demanding invasive procedure for enabling tongue use in TSHCI systems [19]. The two recent TSHCI systems developed at Curtin University employ infrared signals for sensing tongue movement and position. So, they attempt to avoid any demanding physical engagement that would discourage TSHCI systems' application in real life situations.

Following paragraphs analyze the tongue use methods employed in recent Invasive TSHCI (ITSHCI) systems. Infrared signals based Minimally-Invasive TSHCI (MITSCHI) systems developed at Curtin University are then presented and discussed. Finally, challenges and opportunities associated with the development and application of TSHCI systems are discussed.

\section{INVASIVE TSHCI (ITSHCI) SYSTEMS}

For enabling tongue movement/position sensing and using the tongue as an input medium, electrical contact sensors, 
Hall Effect sensors and pressure sensors have been used in many ITSHCI systems. This section introduces some of the prevailing tongue use enabling approaches and discusses their associated problems. Some of the European and US patents are not included in the following discussion for the lack of space. These systems are introduced and discussed in the literature $[10,16]$.

\section{A. Palatal Tongue Controller (PTC)}

Electropalatography (EPG), a technique widely used within the realms of speech therapy, relies on detection of tongue contact with the mouth-roof. The tongue-mouth contact patterns are discovered, displayed and interpreted as a matrix of dots. EPG has been adopted for enabling control of communication devices, wheelchairs and environmental control systems [9]. This self-contained system has a mouth palate connected to a small processing unit. The system is capable of recognizing patterns of tongue movement through detection of tongue-mouth contact. Fig. 1 shows the author's photo montage of the typical mounting arrangement, not necessarily belonging to the PTC under discussion. The electroplatographic system can be employed in three modes. In training mode, PTC would be used to train the user on realizing tongue position and movement. In programming mode, PTC would use patterns of tongue movement for pattern recognition and matching. In user mode, PTC would allow users to access and control external devices and systems. As obvious from Fig. 1, the system requires a demanding and physically invasive procedure to work.

A variation of PTC called InventaidPTC was also designed to control mechanical and electrical functions and control systems [9].

\section{B. Peripheral Device Control Apparatus (PDCA)}

Hall Effect proximity sensors are able to detect changes in a magnetic field caused by the presence of a metallic object. Hall Effect sensors are employed in an ITSHCI apparatus patented in 1994 [10]. Tongue movements are used to control the peripheral devices in this so called PDCA which includes a mouthpiece that consisted of a tongue receptacle. The receptacle is attached to the tongue and is connected to a hollow cylindrical housing. A bar magnet is extended from the receptacle to the inside of the housing. It transmits tongue movements from the receptacle to the housing for quantitative and directional conversion of movements into signals through Hall Effect sensors. The apparatus is able to control various devices including wheelchairs [10].

\section{Inductive Tongue Computer Interface (ITCI)}

An Inductive Tongue Computer Interface (ITCI), proposed in 2006, also required mounting a palatal arrangement on the mouth roof and needed placement of an activation unit on the tongue tip [11]. The ITCI was meant to be used for controlling computers and assistive devices [11]. The ITCI system uses a detection method that is based on the principle of coil induction. The changes in the inductance levels of a coil through movement of a Ferro-magnetic material are exploited. The detected changes in induction level are used to monitor the position/movement of the tongue.

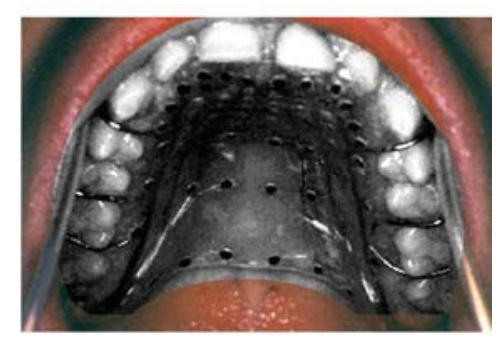

Fig. 1. A montage showing an artistic and symbolic exhibition of a plate and electrodes mounted on a mouth roof.

\section{Tongue Drive Systems (TDS)}

TDS is another ITSHCI system that employs an array of Hall Effect sensors for tracking the tongue movement. It requires a small permanent magnet, housed in a fixture, to be pierced on the tongue. A dental retainer, attached to the teeth, houses the magnetic sensors. Continuous measurements of magnetic field variations provide real-time analog signals to be used by a controller. The controller is a wearable and portable component that remains attached to the body [12].

TDS requires a metallic object to be pierced on the tongue, a dental retainer to be mounted and a controller to be worn. Like the earlier systems, TDS required tongue movement detection through invasive procedures. Thus the usability challenges posed by PTC, PDCA and ITCI were not resolved in TDS. As a permanent magnet has to be pierced onto the tongue, a qualified dentist would be needed to fit the dental retainer. The retainer needs to be customized for each individual's mouth form and geometry. So the system would pose some financial challenges to the patients. The approach may not be financially and physically suitable for all patients. Overall, TDS is a moderately-priced, flexible and easy to operate solution which enables both movement and communication for the patient [8].

An improved version of TDS, called eTDS was introduced later. This improved system, eTDS, would not require a dental retainer to be mounted but would still require a permanent magnet to be attached to the tongue $[13,14]$. eTDS uses a pair of 3-axial magneto-inductive sensor modules driven by a low-power controller. The control unit is wirelessly connected to a nearby computer. Tests suggested that eTDS would track the movements of tongue-mounted permanent magnet and translate them into six pre-defined commands available to a user.

\section{E. Intraoral Tongue Drive Systems (iTDS)}

Intraoral Tongue Drive System (iTDS) evolved through multi-stage improvements in TDS [16]. It uses miniature circuitry so it could be implemented on a printed circuit board that could be fitted inside a dental retainer, similar to the one used in orthodontic procedures [16,17]. iTDS is based on the basic architecture of eTDS but it is a system-on-a-chip that is capable of realizing the magnetic field variations taking place inside the mouth with the help of a set of 3-axial magneto-resistive sensors. A universal interface was designed for iTDS to improve its functionality and usability in demanding and changing environments. 
The systems described in this section evolved during the last two decades or so. As the digital and analog technologies developed, TSHCI systems experienced improvements in architecture, usability related features, functional capabilities and reduction in size. However, they primarily remain invasive and demanding for patients.

\section{Minimally-InVASIVE TSHCI (MITSHCI) SyStemS}

This section provides detailed information on the two infrared signals based MITSHCI systems developed at Curtin University during the last three years.

\section{A. Infrared Tongue Activated Beacon (ITAB)}

An Infrared Tongue Activated Beacon (ITAB) is a device that was designed for receiving emergency signals (input) from the critically ill patient demography. The most important feature of the infrared signal supported ITAB is that it is unobtrusive to both; the patient and the up/down stream health care equipment. Information is exchanged via infrared signals to enable the sensors communicate with the alarm system. The signals won't penetrate any walls and cannot interfere with other health care equipment. The signal receiver module is portable and has the flexibility to allow activation of different emergency systems, which may be required in different patient care situations. ITAB can support wall mounted 'assistance required buttons', alarm and warning systems and carers' pagers. These features make ITAB more suitable for adoption in real life situations [18].

ITAB requires simultaneous and synchronised operation of two sub-systems to function. A transmission sub-system, which upon receipt of a patient's input, can generate an infrared signal for the second sub-system, i.e., a receiver

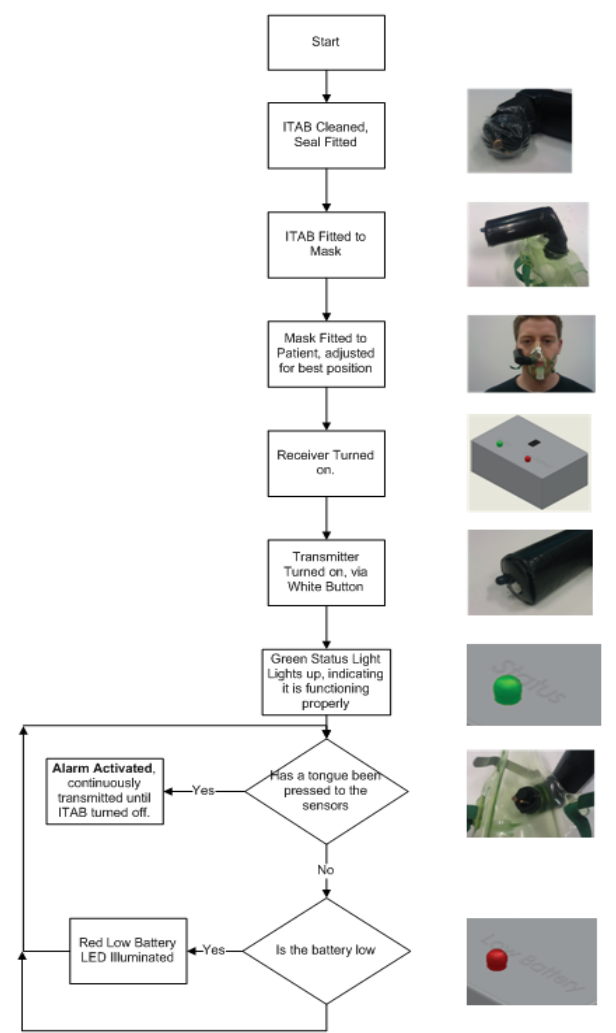

Fig. 2. ITAB's architectural and functional description [19].

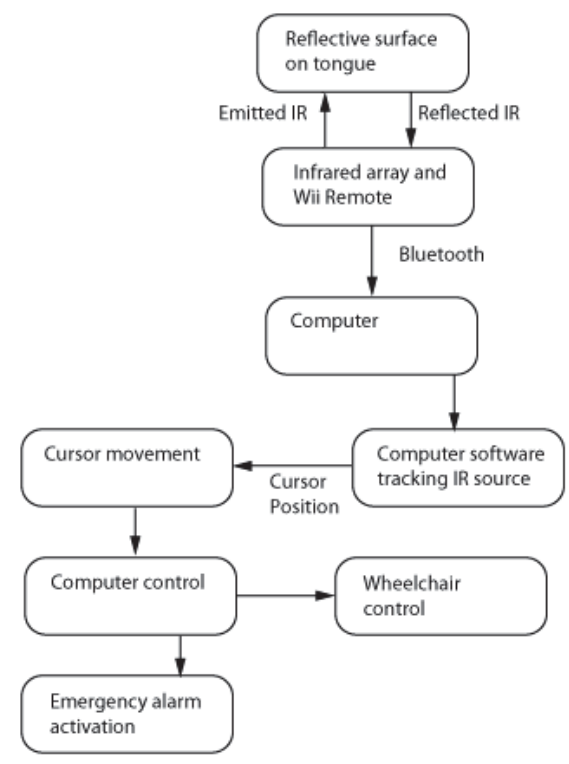

Fig. 3. Architectural and functional description of IRTSHCI system.

component. The receiving sub-system uses the received signal to activate the available alarm or emergency system/s. The implemented system was enclosed in a light weight, chemical resistant plastic casing to be mounted in an oxygen mask like mask that can be attached to a patient's face. The system could also be mounted on a headset. ITAB is designed for mass manufacture and it could be assembled or disassembled easily and swiftly [18]. Fig. 2 presents its architectural and functional descriptions.

\section{B. Infrared Signal Supported HCI System}

ITAB, developed in 2010, had limited aims and scope, meaning it could only be used as an emergency alarm system. In the following attempt, a more versatile and minimallyinvasive infrared TSHCI (IRMITSHCI) system was developed in 2012. It is designed to avoid any intrusive operation and overcome the functional limitations of prevailing ITSHCI systems. The system is focused on; enabling patients communicate with hospital staff (in case of an emergency) and allowing patients to control computers, digital gadgets and wheelchairs. IRMITSHCI design ensures the device is affordable and unobtrusive for the patient. IRMITSHCI device tracks infrared light reflected by the tongue using a Nintendo Wii Remote. The system ensures an acceptable and adoptable MITSHCI system. The functional and architectural descriptions of IRTSHCI system are shown in Fig. 3.

\section{Tongue Movement Sensing}

One of the major tasks in a TSHCI system is the sensing of tongue movement/position and transmitting tongue position to an actuating device or mechanism. Both ITSHCI systems and MITSHCI systems require a signal emitting component to be attached to the tongue for sensing and transmitting tongue movement and position. Various methods and approaches of sensing tongue movement and position are discussed below. 


\section{A. Tongue Position Sensing in Invasive TSHCI Systems}

PTC, an important ITSHCI system discussed in section II relies on the tongue position sensing techniques employed in EPG, a technique widely used in speech therapy. EPG uses an artificial palate, made of acrylic, in which electrodes are embedded between the soft and hard sides of the palate. When the tongue touches any of the electrodes embedded in the palate. Its touch-time and location is recorded, transmitted and displayed. However, EPG poses certain design and usability challenges.

Firstly, mounting the acrylic made palate poses similar problems and limitations an orthodontic brace mounting would pose. Secondly, the patient is expected to have a higher degree of control over tongue movement. Thirdly, a consistent and reliable tongue movement is required for the system to govern the movements of actuating sub-systems. Finally, mounting an acrylic palate and electrodes inside a critically ill patient's mouth may cause fatigue, frustration and a lack of willingness to use the device. Also, training a critically ill patient to position the tongue and control the tongue movement may pose usability challenges.

PDCA, patented in 1994 in the US, requires a bar magnet to be attached to the tongue. It is complimented with a funnel-shaped mouth piece. The mouth piece, uses the embedded Hall sensors and the bar magnet on the tongue, to detect the direction and magnitude of tongue movement and tongue displacement with respect to the centre of the mouth piece. There are several problems in using PDCA. Firstly, it has an inconvenient bar magnet-mouth piece arrangement, less acceptable to an already distressed patient demography (e.g. Quadriplegics). Secondly, PDCA is hard-connected (using wires) to the main processor and so poses movement challenges. Thirdly, the system would physically engage the tongue and the oral cavity of a user. Though the mounting arrangement of PDCA is much different than that used in PTC; yet, the implementation is physically invasive and may cause fatigue and frustration to the patients. Also, a patient is required to develop tongue-movement control skills for aptly operating the apparatus.

ITCI, introduced in 2006, also requires mounting a palatal arrangement inside the mouth. Furthermore, an activation unit is fitted on the tongue tip. Hence, ITCI poses all the limitations that PTC would pose.

The different versions of TDS discussed in section II also pose similar challenges and limitations. Like the earlier systems, TDS, eTDS and iTDS require tongue movement detection through invasive procedures. Thus the usability challenges posed by PTC, PDCA and ITCI were not resolved in TDS. As the permanent magnet has to be pierced onto the tongue, a qualified dentist would be needed to fit the dental retainer. The retainer would usually be customized for each individual's mouth. So the system would also pose financial challenges to the patients [15]. The improved TDS, introduced as eTDS, would not require a dental retainer to be mounted but would still require a permanent magnet to be attached to the tongue [12,13]. The following version of TDS, called iTDS is based on the basic architecture of eTDS though it is a system-on-a-chip and is capable of realizing the

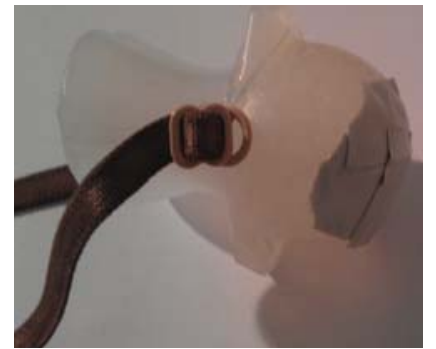

Fig. 4. Silicone tongue device. The reflective tape is applied to the far side of the device

magnetic field variations taking place inside the mouth with the help of an externally mounted set of 3-axial magneto resistive sensors.

\section{B. Tongue Position Sensing in MITSHCI systems}

Tongue input in the Infrared Tongue Activated Beacon (ITAB) is limited to activating an alarm system. Only a low pressing force would suit the patient demography. As the patient presses the button, a Light Dependent Resistor (LDR) is activated. A supporting sensor, a temperature dependent resistor (a thermistor) added to the circuit, activates the alarm system in dark conditions and during night hours. The patient is expected to be at least $10{ }^{\circ} \mathrm{C}$ warmer than the ambient environment. Even when the patient comes in contact with the thermistor for 1-2 seconds, in dark conditions, the alarm system is activate-able. Hence, the sensor array includes a push button, a LDR and a thermistor, all mounted in a very close proximity. The system can be activated when any two of the three sensors find positive signals from the patient. A low-cost and efficient PICAXE $8 \mathrm{~mm}$ microcontroller was used to perform the logic operations. The SIRC protocols were used for transmitting signals from the sensing circuit to the receiving (alarm) circuit. A 12-bit SIRC was required in ITAB.

The input sub-system was enclosed in a light weight case strong enough to hold the components and survive a drop to the floor. The case needs to withstand saliva having a $\mathrm{pH}$ range of $6.0-7.4[20,21]$. The top 3 images on the right side in Fig. 2 show the case, its mounted position (with an oxygen mask), and the input system being used. It is obvious from Fig. 2 that the tongue sensing system is minimally invasive and would not require an impaired patient to go through any painful or expensive process.

A BRM-15S8-11 compact receiver, common in remote sensing applications, receives the signals. Its peak wavelength (940 n-meters) matches that of the infrared Light Emitting Diode (LED). With a built-in band pass to reject the ambient light, it is capable of receiving $38 \mathrm{kHz}$ SIRC signals.

The infrared minimally-invasive human computer interaction (IRMITSHCI) system avoids any excessive use of intrusive methods/means for enabling interaction with the control module of the system. In order to work in a suitable range, a reflective medium is used to reflect infrared light to the Wii remote's infrared camera [16,17]. The employed reflective tape is positioned on the tongue as it meets the following criteria:

(a) The medium has to be as less obtrusive as possible; 


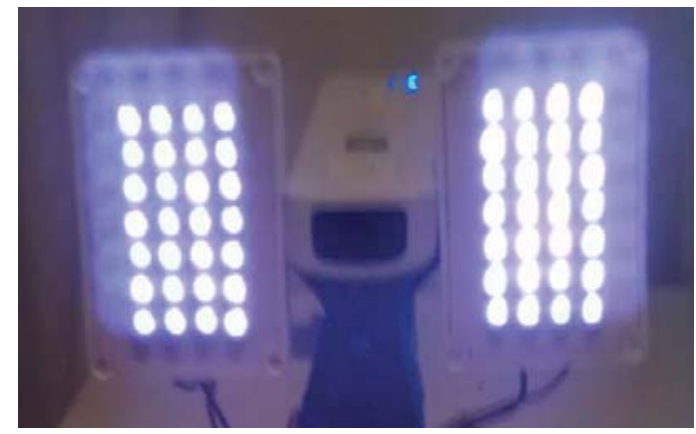

Fig. 5. The Infrared Array System

(b) The medium is wearable by impaired and ill patients for extended periods of time; and

(c) The medium must be easy to clean.

A silicone tongue device, usually worn by sleep apnea patients, was considered to be suitable for this IRMITSHCI system as the device can be easily removed by a patient. Mounting the reflective tape is an easy to implement and low cost solution. The device, shown in Fig. 4 can be comfortably worn for long periods of time. Since it is made of medical grade silicone, it is easy to clean and sterilize.

Patients' input, via the tongue-attached device is sent to a remote Wii infrared (IR) camera. In designing the system, it was assumed that the tongue tracking system will be used within a distance of about $1.5 \mathrm{~m}$, the maximum recommended usable range for the device is $5 \mathrm{~m}$. The band pass filter built into the Wii remote camera restricts detecting light sources outside the desired wavelength. The infrared light travels to the patient and back to the infrared camera. Hence, an infrared array was designed to output enough light when the system is activated. The selected array, shown in Fig. 5 is a 4 $x 7$ LED-array and the LED's have a wavelength of $875 \mathrm{~nm}$. The angle of half intensity of the LED was $\pm 12^{\circ}$. This enabled the array to easily achieve an adequate range and wider FoV angles. A $13.5 \mathrm{~V}$ DC supply outputting a total of $800 \mathrm{~mA}$ was used to ensure that the LED's were outputting the required light intensity. A simple bi-directional switch was added in order to turn on and off the connection to the supply.

The Wii remote camera outputs are processed in two stages. First, a connection between the remote and the computer is made via Bluetooth. Then, a computer program transforms the raw output (infrared data) into usable output signals. A Bluetooth dongle is connected to the computer running under Windows 7 OS. The Bluetooth software recognizes the presence of the remote and connects upon a simple mouse click. Once the connection is established, a software program handles the inputs (coming from the Wii remote's infrared camera) and translates the incoming data into both cursor movement and output signals needed to control any wheelchair type device. FreeTrack, a multipurpose optical motion tracking software was employed to accurately track sources of infrared light. By using FreeTrack, it was easy to track the tongue tip movements and provide patients with the required degree of control over a mouse cursor. The cursor control opens the door to a variety of communication and transport possibilities for patients. For

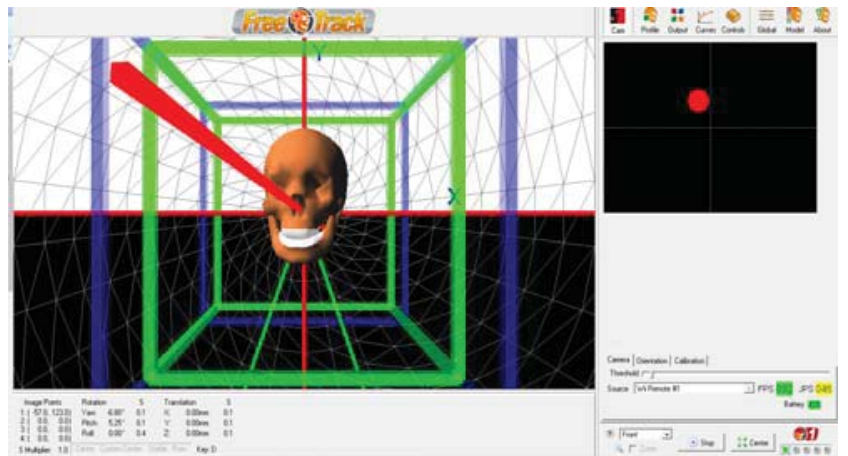

Fig. 6. FreeTrack operation, showing the movement of an object in an off center position

achieving this goal, FreeTrack was used together with a dwell clicking program to provide the mouse clicking functionality.

The mouse cursor navigation task was accomplished by first generating a profile within FreeTrack which could be used for mouse control. Profiles within FreeTrack allow for the system to incorporate the needs of each individual patient; making it simple to quickly adapt to the needs of different people. FreeTrack uses threshold control in order to eliminate unwanted sources of light interference. The threshold level in IRMITSHCI was set by adjusting the threshold control bar until only the infrared blob, being reflected back by the reflective tape, was visible on the FreeTrack blob tracking interface. FreeTrack would take the current position of the tongue to be the center position for all future output purposes. Once the threshold level and center position are set, the mouse output, together with the auto pan option can be selected within the program. The auto pan function allows the mouse cursor to move across the entirety of the screen, it works by initially detecting that the tongue is located away from the center point. The mouse cursor will then continue to auto pan in the same direction that the tongue is from the center point, when the tongue is brought back to the center point the cursor will stop. After a set period of time, a dwell clicking program, Dwell Clicker 2, will initiate a mouse click. Dwell clicking programs are commonly used by people suffering from repetitive strain injuries. It provides full functionality for the user in the sense that it can achieve leftclick, right-click, double-click or drag. Click options can easily be changed through the use of large usable icons.

\section{Tongue Movement/ Position SEnsing AND USABILITY OF TSHCI SYSTEMS}

Human factors such as: Tongue movement and location of sensors; the method/procedure involved in mounting them; physical dimensions, size and physical location of the mounted hardware would affect device acceptability by the carers and patients [22]. Therefore, successful deployment of a well-designed TSHCI system would always depend on the outcome of the device usability evaluation. An empirical set of TSHCI system evaluation criteria is not yet available. It is therefore imperative for the TSHCI system designers to develop an ad hoc set of usability evaluation criteria and apply the same for evaluating any existing/future TSHCI systems. Based on an extensive literature review [23-26], important aspects of TSHCI system design can be evaluated using the set of eight questions presented in Table I. Each 
TABLE I. USABILITY ASPECTS AND EVALUATION QUESTIONS

\begin{tabular}{|l|l|}
\hline \multicolumn{1}{|c|}{$\begin{array}{c}\text { Usability } \\
\text { Aspect }\end{array}$} & \multicolumn{1}{c|}{ Evaluation Question } \\
\hline Acceptability & Would a patient/carer/doctor like to use the system? \\
\hline Functionality & $\begin{array}{l}\text { Would a patient/carer/doctor find the system complex } \\
\text { and difficult to use? }\end{array}$ \\
\hline Portability & $\begin{array}{l}\text { In order to deploy/use the system, would a } \\
\text { patient/carer/ doctor need help from a support staff? }\end{array}$ \\
\hline Usefulness & Does the system provide all the required functions? \\
\hline Efficiency & Are all the required functions well-integrated? \\
\hline Effectiveness & $\begin{array}{l}\text { Would a patient/carer/doctor find it easy to learn and } \\
\text { use the system? }\end{array}$ \\
\hline Satisfaction & $\begin{array}{l}\text { Would a patient/carer/doctor feel confident using the } \\
\text { system? }\end{array}$ \\
\hline Acceptability & $\begin{array}{l}\text { Is the system affordable for a large proportion of } \\
\text { patient demography? }\end{array}$ \\
\hline
\end{tabular}

question addresses a certain aspect of TSHCI system design which is also shown in the table.

Please note that the evaluation questions require a twoprong evaluation of the system. The system must be evaluated by carers/doctors. The system also needs to be evaluated by the patient demography. Without a rigorous two-prong evaluation, the real-life success of a TSHCI system should not be assumed.

\section{CONCLUSION}

Recent works have shown the efficacy of the tongue as a medium of input in TSHCI systems. However, designing an effective and widely acceptable TSHCI system would require incorporation of several important features. Some of these features like minimal-intrusion, input signal conditioning efficiency, system reliability, effectiveness of the control strategy, and ease of learning and using the system are highly important. Methods of incorporating these features can be used for dividing the existing TSHCI systems into invasive and minimally-invasive systems. It was realized that methods and techniques of realizing and sensing tongue movement and position determine the level of invasiveness in a TSHCI system. These techniques, as employed in important TSHCI systems, were examined and analyzed in this paper. Several previously developed TSHCI systems and two infrared minimally-invasive human computer interaction (IRMITSHCI) systems, developed at Curtin University, were presented and discussed to examine ways of minimizing the TSHCI systems' intrusiveness. Important criteria required to evaluate the usability of TSHCI systems were also proposed.

\section{REFERENCES}

[1] P. Bach-y-Rita, K.A. Kaczmarek, M.E. Tyler and J. Garcia-Lara, "Form perception with a 49-point electrotectile stimulus array on the tongue: A technical note," Journal of Rehabilitation Research and Development, vol. 35, no. 4, 1998.

[2] P. Bach-y-Rita, "Physiological considerations in sensory enhancement and substitution,” Europa Medicophysica, Vol. 25, pp. 107-128, 1989.

[3] P. Bach-y-Rita and S.W. Kercel, "Sensory substitution and the humanmachine interface," Trends in Cognitive Science, Vol. 7, no. 12, 2003.

[4] N. Vuillerme, N. Pinsault, O. Chenu, A. Fleury, Y. Payan, J. Demongeot, "A wireless embedded tongue tactile biofeedback system for balance control,” Passive and Mobile Computing, vol. 5, pp. 268275, 2009.

[5] A. Dix, J. Finlay, G. D. Abowd, and R. Beale, Human Computer Interaction. London: Pearson Education - Prantice Hall, 2004.

[6] R. Sharma, V. I. Pavlovic, and T. S. Huang, "Toward multimodal human-computer interface," Proceedings of IEEE, vol. 68, pp. 853869, 1998.

[7] A. Jaimes and N. Sebe, "Multimodal Human Computer Interaction: A Survey," in Computer Vision in Human Computer Interaction: Lecture Notes in Computer Science. vol. 3766, ed: Springer Berlin Heidelberg, 2005, pp. 1-15.

[8] C. Lau and S. O'Leary, "Comparison of Computer Interface Devices for Persons With Severe Physical Disabilities," American Journal of Occupational Therapy, vol. 47, pp. 1022-1030, 1993.

[9] C. Clayton, R. G. S. Platts, M. Steinberg, and J. R. Hennequin, "Palatal Tongue Controller," Journal of Microcomputer Applications, vol. 15, pp. 9-12, 1992.

[10] N. Buchhold, "Apparatus for controlling peripheral devices through tongue movement, and method of processing control signals," US Patent US5460186 A 1995.

[11] N. S. A. Strujic, "An Inductive Tongue Computer Interface for Control of Computers and assistive Devices," IEEE Transactions on Biomedical Engineering, vol. 53, 2006.

[12] G. Krishnamorthy and M. Ghovanloo, "A Tongue Operated Magnetic Sensor Based Wireless Assistive Technology for People with Severe Disabilities," in Proceedings of ICAS 2006, IEEE International Symposium on Circuits and Systems (ISCAS), Greece 2006.

[13] X. Huo, J. Wang, and M. Ghovanloo, "A Magneto-Inductive Sensor Based Wireless Tongue-Computer Interface," IEEE Transactions on Neural Systems and Rehabilitation Engineering, vol. 16, pp. 497-504, October 20082008.

[14] X. Huo and M. Ghovanloo, "Using unconstrained Tongue Motion as an Alternative Control Mechanism for Wheeled Mobility," IEEE Transactions on Biomedical Engineering, vol. 56, pp. 1719-1726, June 20092009.

[15] H. Park, M. Kiani, H.-M. Lee, J. Kim, J. Block, B. Gosselin, and M. Ghovanloo, "A Wireless Magnetoresistive Sensing System for an Intrroral Tongue-Computer Interface," IEEE Transactions on Biomedical Circuits and Systems, vol. 6, pp. 571-585, December 2012 2012.

[16] P. S. Sheriff and R. J. York, "Oral machine controller," US Patent US4728812 A, 1988.

[17] P. Hangue, K. Jeonghee, and M. Ghovanloo, "Intraoral Tongue Drive System Demonstration," in 2012 Biomedical Circuits and Systems Conference (BioCAS), Taiwan, 2012.

[18] J. Kim, H. Park, and M. Ghovanloo, "Tongue-Operated Assistive Technology with Access to Common Smartphone Applications via Bluetooth Link," in 34th International Conference of the IEEE EMBS, San Diego, CA, 2012.

[19] S. Sandy and M. M. Khan, "A Tongue-Activated Emergency Beacon for Immobile Patients," in IEEE-EMBS International Conference on Biomedical and Health Informatics (BHI 2012), Shenzhen, China, 2012.

[20] A. C. Guyton, Textbook of Medical Physiology.: Saunders, 1991.

[21] H. C. Crow and J. A. Ship, "Tongue strength and endurance in different aged individuals," The Journals of Gerontology Series A Biological Sciences and Medical Sciences, vol. 51, pp. M247-50, 1996.

[22] G. Ginsburg, "Human Factors Engineering: A tool for medical device evaluation in hospital procurement decision-making," Journal of Biomedical Informatics, vol. 38, pp. 213-219, June 20052005.

[23] R. J. Fairbanks and S. Caplan, "Poor interface design and lack of usability testing facilitate medical error," Joint Commission journal on quality and safety, vol. 30, pp. 579-584, October 20042004.

[24] J. M. C. Bastien, "Usability testing: a review of some methodological and technical aspects of the method," International journal of medical informatics, vol. 79, pp. e18-e23, 2010.

[25] M. W. M. Jaspers, "A comparison of usability methods for testing interactive health technologies: Methodological aspects and empirical evidence," International journal of medical informatics, vol. 78, pp. 340-353, 2009.

[26] E. Liljegren, "Usability in a medical technology context assessment of methods for usability evaluation of medical equipment," International Journal of Industrial Ergonomics, vol. 36, pp. 345-352, 2006. 